\title{
Preface to the special issue: Novel functionalities of tungsten-related materials
}

\section{Shu Yin ${ }^{1}$}

(c) The Nonferrous Metals Society of China 2020

Tungsten is one of the most important strategic resources of rare metallic elements. Tungsten journal focuses on the tungsten and related elements, including molybdenum, cobalt, vanadium, tantalum, niobium, rhenium, together with their compounds. Traditional tungsten-based materials have already shown a wide range of applications such as filaments of a bulb, high hardness alloys, bullets for the anti-tank and anti-ship. Recently, many researchers have also paid their attentions to the new functionality and novel applications of tungsten-based materials. Many researches focus on the synthesis processes of tungsten-related materials, together with the precise control of their crystalline phases and macro/ micro/nano-structures, surface modification, etc. Many novel syntheses and characterizations are carried out, discovering many novel applications. Tungsten-related materials show exciting progress on life science, optical materials, photovoltaic materials, energy storage materials, catalysts, etc.

The present special issue focuses on novel functionalities of tungsten-related materials, consisting of seven contributions with two review papers and five original research articles. A review paper reports the progress on tungsten-based photocatalyst materials with unique ultraviolet-, visible- and near-infrared-light-induced photocatalytic activities (Y Wang, et al., Lanzhou Univ., China). One original paper investigates the photoluminescence property of $\mathrm{ZnWO}_{4}$, which has various morphologies such as nanocrystals, nanorods, nanoplates and spheroidal clusters (JG Li, et al., National Institute for Materials Science (NIMS), Japan). Besides above tungsten-based compounds, some articles consisted of molybdenum, vanadium and niobium elements are also included in this special issue. A short review paper reports the surface modification of molybdenum oxide nanowires, and their high performances on the high energy storage. (W Dong, et al., Beijing Univ. Sci. Tech., China). Anion-doped vanadium dioxide and its composite thin films show an excellent thermal chromic property, indicating its potential application for the smart window coating (the cover image of the special issue, $\mathrm{S}$ Yin, et al., Tohoku Univ., Japan). $\mathrm{Li}_{3} \mathrm{VO}_{4}$ possesses the excellent specific capacity, suitable discharge voltage and moderate volume change upon charge/discharge. A partial surface phase transformation leads to the formation of the conductive $\mathrm{LiVO}_{2}$ layer on the electrode material $\mathrm{Li}_{3} \mathrm{VO}_{4}$, resulting in its outstanding rate performance and longtime cycle stability for next-generation lithium-ion batteries. (G Li, et al., Jilin Univ., China). On the other hand, the $4 d-4 f$ transition metal $\mathrm{La}-\mathrm{Nb}-\mathrm{O}$ compounds show the great potential for fast lithium-ion energy storage. The related research provides some structure clues for the design of electrode materials for fast lithium storage (D Xue et al., Changchun Institute of Applied Chemistry (CICA), Chinese Academy of Sciences (CAS), China). Also, a high-temperature alloy GH4169 containing molybdenum and niobium elements might be fabricated with controllable porosity and mechanical properties using the laser melting deposition (LMD) technique, showing the potential application on 3D printing. (B Zhang, et al., Northeastern Univ., China).

Novel functionality of tungsten-related materials is a wide range topic. Although the present special issue can only cover the partial information of the related research area, I hope and believe that this special issue could provide a chance for researchers/scientists to understand the related innovative research trend and ideas in the research field. Also, I would like to appreciate all the authors, reviewers, editors, and publishing staff for their great efforts and contributions for the publication of this special issue.

Shu Yin

yin.shu.b5@tohoku.ac.jp

1 Institute of Multidisciplinary Research for Advanced Materials, Tohoku University, Sendai 980-8577, Japan 


\section{S. Yin}

Shu Yin

Dec 15, 2019

Publisher's Note Springer Nature remains neutral with regard to jurisdictional claims in published maps and institutional affiliations.

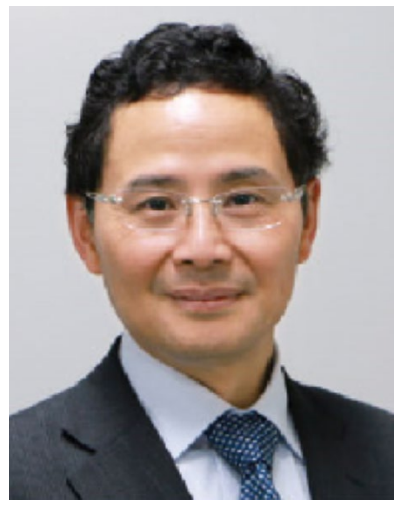

Prof. Shu Yin received his Ph.D. degree in applied chemistry from Tohoku University (research period shortened) in 1999. He has been a full-time professor in Tohoku University from 2016 and is also an affiliate professor of eight Chinese universities/ institutes, including Lanzhou University, Dalian University of Technology, Guangxi Research Institute of Chemical Industry, Huaqiao University, Taiyuan University of Technology,
Sichuan University, Beijing University of Technology, University of Science and Technology Beijing, State Key Laboratory of Rare Earth Resource Utilization. He is the chair of Japan Chapter, Functional Materials Society, and also acts as the symposium chair of some international conferences such as ISFM2011 and ISHA2018. He is an editor of some scientific journals such as Ceram. Int., Funct. Mater. Lett., Nanoscience, Tungsten, and Rare Metals. He has authored more than 450 original research papers, contributed 30 book chapters/review papers and 23 patents. His research papers were cited more than $\mathbf{1 2 4 7 0}$ times and showed a citation $\boldsymbol{h}$-index of $\mathbf{6 2}$ (Google citation). His research interests include morphological control of nanostructured oxides/nitrides, photocatalytic materials, UV/IR-shielding materials, gas sensing materials, hydrothermal/solvothermal process, soft chemical synthesis. 